\title{
An Alternative Approach to Energy Eigenvalue Problems of Anharmonic Potentials
}

\author{
Okan Ozer and Halide Koklu \\ Department of Engineering Physics, Engineering Faculty, University of Gaziantep, 27310 Gaziantep, Turkey \\ Correspondence should be addressed to Okan Ozer; ozer@gantep.edu.tr
}

Received 25 May 2014; Accepted 22 July 2014; Published 27 August 2014

Academic Editor: Andrei D. Mironov

Copyright ( 2014 O. Ozer and H. Koklu. This is an open access article distributed under the Creative Commons Attribution License, which permits unrestricted use, distribution, and reproduction in any medium, provided the original work is properly cited.

Energy eigenvalues of quartic and sextic type anharmonic potentials are obtained by using an alternative method called asymptotic Taylor expansion method (ATEM) which is an approximate approach based on the asymptotic Taylor series expansion of a function. It is shown that the energy eigenvalues found by ATEM are in excellent agreement with the existing results.

\section{Introduction}

It is well known that the exact solution of Schrödinger equation is only possible for very few numbers of potentials and it is required to apply the numerical methods or some approximate schemes in most quantum mechanical systems. In the literature, there are many studies for this purpose such as numerical calculation $[1,2]$, the perturbation [3], the variational $[4]$, the $\mathrm{WKB}[5,6]$, the shifted $1 / N$ expansion $[7,8]$, the Nikiforov-Uvarov (NU) [9], and the supersymmetry (SUSY) [10] to find the approximate solutions of the potentials that are not exactly solvable. In the last decades, the study of anharmonic oscillator potentials (such as quartic and sextic anharmonic oscillators) has been taking attention because they are open for the theoretical perception of some recently discovered phenomena in different branches of physics [11-13]. On the other hand, these potentials are not exactly solvable which make them very popular for examining the validity of any approach [14-17]. In all these attempts, it is merely required to get a relatively effective and simple approach that gives the energy eigenvalues-and eigenfunctions-to a high degree of accuracy. In this study, an efficient method called asymptotic Taylor expansion method (ATEM) is applied to compute the energy eigenvalues of both the quartic and the sextic anharmonic oscillator potentials in one dimension.
The ATEM based on the Taylor series expansion is proposed by Koç and Sayın [18]. It is claimed that one can easily apply ATEM to solve second-order differential equations by introducing a simple code in Mathematica [19] computer program. Therefore, it is focused on the solution of the eigenvalue problems of anharmonic oscillator potentials by using ATEM in this paper.

The organization of the paper is as follows. In Section 2, a brief outline of the method ATEM is presented. In Section 3, we obtain numerically the eigenenergies and make comparison with other existing results. Finally, Section 4 is devoted to a conclusion.

\section{Asymptotic Taylor Expansion Method}

Following the notation in [18], one can consider the Taylor series expansion of a function $f(x)$ about the point $a$ :

$$
\begin{aligned}
f(x)= & f(a)+(x-a) f^{\prime}(a)+\frac{1}{2}(x-a)^{2} f^{\prime \prime}(a) \\
& +\frac{1}{6}(x-a)^{3} f^{(3)}(a)+\cdots \\
= & \sum_{n=0}^{\infty} \frac{(x-a)^{n}}{n !} f^{(n)}(a)
\end{aligned}
$$


where $f^{(n)}(a)$ is the $n$th derivative of the function at $a$. If $a=0$, then it is called Maclaurin's series and given by

$$
\begin{aligned}
f(x) & =f(0)+x f^{\prime}(0)+\frac{1}{2} x^{2} f^{\prime \prime}(0)+\frac{1}{6} x^{3} f^{(3)}(0)+\cdots \\
& =\sum_{n=0}^{\infty} \frac{x^{n}}{n !} f^{(n)}(0) .
\end{aligned}
$$

It is claimed in [18] that one can construct a method to solve the second-order linear differential equations in the form of

$$
f^{\prime \prime}(x)=p_{0}(x) f^{\prime}(x)+q_{0}(x) f(x) .
$$

By differentiating (3) with respect to $x$, one can get the higher order derivatives of $f(x)$ in terms of $f(x)$ and $f^{\prime}(x)$. Thus, one obtains

$$
f^{(n+2)}(x)=p_{n}(x) f^{\prime}(x)+q_{n}(x) f(x),
$$

where

$$
\begin{aligned}
& p_{n}(x)=p_{0}(x) p_{n-1}(x)+p_{n-1}^{\prime}(x)+q_{n-1}(x), \\
& q_{n}(x)=q_{0}(x) p_{n-1}(x)+q_{n-1}^{\prime}(x) .
\end{aligned}
$$

At this point, it is seen that the eigenvalues and eigenfunctions of the Schrödinger-type equations can efficiently be obtained by using ATEM. To this end, the recurrence relations (5) allow one to get analytical or numerical solution of (3) under some certain conditions. After substituting (5) into (1) and obeying the termination condition $n=m$ of the eigenfunction $f(x)$ in the bound-state quantum mechanical systems (the reader is addressed to [18]), one can finally write

$$
\begin{aligned}
q_{m}(0) f(0)+p_{m}(0) f^{\prime}(0) & =0, \\
q_{m-1}(0) f(0)+p_{m-1}(0) f^{\prime}(0) & =0
\end{aligned}
$$

and then eliminating $f(0)$ and $f^{\prime}(0)$ one gets

$$
q_{m}(0) p_{m-1}(0)-p_{m}(0) q_{m-1}(0)=0
$$

which will involve a parameter related to eigenvalues of the potential in the study.

It is seen that the method ATEM is iterative and iteration number is given by $m$. It is proposed that the method can be applied to the Schrödinger equation, with any type of potentials, as follows. Using a Mathematica computer program, one can repeat the calculation of the eigenvalues for different values of iteration number such as $m=10,20,30, \ldots$, till desired digits. When the eigenvalues reach their asymptotic value, then one can choose the corresponding $m$ and truncate the iteration for next calculations. For instance, if one obtains the eigenvalues for desired digits when $m=80$, then the first few eigenvalues-first eight-energy state, for example-will automatically reach their asymptotic values [20].

\section{Applications}

We consider the one-dimensional quartic double well potential as a first application:

$$
V(x)=x^{4}-\gamma x^{2}, \quad \gamma>0 .
$$

This potential has great importance in many branches of physics such as molecular vibrations [21], solid state physics $[22,23]$, quantum field theories [24], and quantum chromodynamics [25]. The Schrödinger equation is written as

$$
\left(-\frac{d^{2}}{d x^{2}}+x^{4}-\gamma x^{2}\right) \psi(x)=E \psi(x),
$$

where $\hbar^{2}=2 m=1$. To apply ATEM, now we introduce an ansatz wavefunction as the asymptotic solutions of (9):

$$
\psi(x)=e^{-\alpha\left(x^{2} / 2\right)-\beta\left(x^{4} / 4\right)} f(x)
$$

and (9) can now be written as

$$
\begin{aligned}
L(x)= & -f^{\prime \prime}(x)+2\left(\alpha x+\beta x^{3}\right) f^{\prime}(x) \\
& +\left(\alpha-E+\left(3 \beta-\alpha^{2}-\gamma\right) x^{2}+(1-2 \alpha \beta) x^{4}-\beta^{2} x^{6}\right) \\
& \times f(x)=0 .
\end{aligned}
$$

Comparing (3) and (11) one can deduce that

$$
\begin{gathered}
p_{0}(x)=2\left(\alpha x+\beta x^{3}\right), \\
q_{0}(x)=\alpha-E+\left(3 \beta-\alpha^{2}-\gamma\right) x^{2}+(1-2 \alpha \beta) x^{4}-\beta^{2} x^{6} .
\end{gathered}
$$

Before proceeding ATEM, we note here some points about the ansatz wave function and the selection of numerical values of adjustable parameters $\alpha$ and $\beta$; to satisfy the quantum mechanical postulates in the limit of large $x$ for the bound systems, the asymptotic solutions of (9) can be taken as the multiplication of any power of $x$ with a decreasing Gaussian-type function. Therefore, we suggest the ansatz wave function in the form of (10). Additionally, there is strong relation among the wave function and the potential parameters of $\alpha, \beta$, and $\gamma$, respectively, in the solution of (9). Since the exact analytical solution of the potential in (9) cannot be obtained, then one can look for the effective partner potential solutions; since we introduce (10), then the superpotential function for the partner potentials by supersymmetric quantum mechanics (SUSYQM) [26] is given by

$$
W(x)=-\frac{\psi^{\prime}(x)}{\psi(x)},
$$

and the partner potentials $V_{ \pm}(x)$ are defined as

$$
V_{ \pm}(x)=W^{2}(x) \pm W^{\prime}(x) .
$$

Since the superpotential $W(x)$ obtained from (10) is

$$
W(x)=\alpha x+\beta x^{3}
$$

then the partner potential $V_{-}(x)$ is obtained as

$$
V_{-}(x)=\left(\alpha^{2}-3 \beta\right) x^{2}+2 \alpha \beta x^{4}+\beta^{2} x^{6}-\alpha .
$$


It is observed that the potential (16) has one minimum if $\alpha^{2}>3 \beta$. Searching for this one-minimum case, we select the adjustable parameters as $\alpha=2$ and $\beta=1$ in this study. Since the iteration number $m$ is desired to be as much low as possible, then one has to search for the optimum iteration number in the calculation of eigenvalues of potential in (9). By setting $\gamma=2, \alpha=2$, and $\beta=1$, we search for the optimum iteration number $m$ by comparing the results of ATEM with the known exact value of third excited state, $E_{n=3}^{\text {exact }}=8.33293$, in Table 1. We also obtain the percent errors and then set $m=80$ to truncate the iteration in the following calculations. It is seen that the term asymptotic means the ATEM value approaching to a given value as the iteration number tends to infinity.

We present our results carried out for a range of $\gamma$ values in Table 2 and Table 3 with 7 significant digits and they are compared with those of variational supersymmetric approach by [27] and the ones computed numerically by [28]. In our calculations, we set $m=80, \alpha=2$, and $\beta=1$. We observe that the results by ATEM are in very good agreement with numerical ones of [28]. In particular, for $\gamma<1$, the errors are less than $1 \%$ in all cases.

As a second application, we consider the energy eigenvalues of the quasi-exactly solvable sextic anharmonic oscillator potentials:

$$
V(x)=x^{6}-(8 j+3) x^{2}, \quad j=0, \frac{1}{2}, 1, \frac{3}{2}, \ldots
$$

Since only $(2 j+1)$ energy eigenvalues can be obtained analytically while the other levels remain unknown, these potentials are classified as quasi-exactly solvable potentials $[29,30]$. Following the same procedure given above and the ansatz wave function in (10) by the same numerical values of $m=80, \alpha=2$, and $\beta=1$, one obtains

$$
\begin{aligned}
L(x)= & -f^{\prime \prime}(x)+2\left(2 x+2 x^{3}\right) f^{\prime}(x) \\
& +\left(2-E-(4+8 j) x^{2}-4 x^{4}\right) f(x)=0 .
\end{aligned}
$$

We present and compare our results in Table 4 for $j=$ $0,1 / 2,1,3 / 2$. It is seen from Table 4 that the eigenvalues obtained by the ATEM are acceptably in good agreement with the exact numerical results [31]. We also present the percent errors. It is observed that the errors are less than $1 \%$ except the highest energy state-in this study-for $j=0,1 / 2,1$ values.

As a last example, we consider the quasi-exactly solvable potential [6] given as

$$
V(x)=x^{6}+10 x^{4}+10 x^{2} .
$$

Using the ansatz wave function of the form defined in (10) and following the same procedure given above for the same numerical values of $m=80, \alpha=2$, and $\beta=1$, one obtains

$$
\begin{aligned}
L(x)= & -f^{\prime \prime}(x)+\left(4 x+2 x^{3}\right) f^{\prime}(x) \\
& +\left(2-E+9 x^{2}+6 x^{4}\right) f(x)=0 .
\end{aligned}
$$

We present and compare our results for $n=0,2,4$ energy states in Table 5. It is seen that ATEM reproduces excellent results consistent with the existing literature.
TABLE 1: Comparison of third excited state eigenvalue of the quartic potential in (9) by means of ATEM for $\gamma=2, a=2$, and $b=1$ and selection of optimum value for iteration number $m$ due to percent error calculations.

\begin{tabular}{lcc}
\hline$m$ & $E_{\text {ATEM }}$ & Error (\%) \\
\hline 10 & 27.22914 & 226.766 \\
20 & 10.70786 & 28.500 \\
30 & 9.167173 & 10.011 \\
40 & 8.612519 & 3.355 \\
50 & 8.421598 & 1.064 \\
60 & 8.359757 & 0.322 \\
70 & 8.340775 & 0.094 \\
80 & 8.335161 & 0.027 \\
90 & 8.333531 & 0.007 \\
100 & 8.333059 & 0.002 \\
\hline & $E_{n=3}^{\text {exact }}=8.33293$ & \\
\hline
\end{tabular}

\section{Conclusion}

We have applied relatively simple and efficient method for calculating the energy eigenvalues of some type of onedimensional anharmonic oscillator potentials. The method is based upon the asymptotic Taylor series expansion of a function. It is shown that the optimal truncation of the Taylor series reproduces the numerical results for eigenvalues with higher accuracy. The algorithm constructed for a computer system using symbolic or numerical calculation is relatively simple in ATEM.

It is clear that the determination of the adjustable parameters $\alpha$ and $\beta$ directly affects the iteration number $m$. Therefore, the efficiency of the method is then dependent on these parameters. Since we set $m=80$ by choosing $\alpha=2$ and $\beta=1$, then the iteration number may seem to be not low enough. One can choose different $\alpha$ and $\beta$ values obeying the constraint given in text; then the iteration number is expected to get lower values. On the other hand, one can also investigate the best approximate values of the parameters $\alpha$ and $\beta$ by searching the minimum expectation value of energy that satisfies (9), for example, for the ground state. Since we focus on the application of ATEM for the determination of the energy eigenvalues by choosing $\alpha$ and $\beta$ values given above, it is thought in our calculations that the percent error ranges for the potentials in this study are acceptable for the selected values of $\alpha$ and $\beta$ parameters, by satisfying the minimum-case condition of supersymmetric partner-like potentials.

It is believed that the simplicity and mathematical facility of the method suggested here can be useful to the treatment of the Schrödinger equation including large class of potentials. For example, the method can be used for other unidimensional potentials and for isotropic potentials in three dimensions. Additionally, one can focus on the potential function given in (8) to search for energy splitting in the wells for $\gamma$ value being large enough [33]. Studies along this line are in progress. 
TABLE 2: Energy eigenvalues for the potential in (9) with different values of $\gamma \cdot E_{0,1}(V)$ and $E_{0,1}(N)$ show the values obtained by variational supersymmetric method and numerical integration, respectively. The percent errors for ATEM are also shown in the fifth and ninth columns.

\begin{tabular}{lcccccccc}
\hline$\gamma$ & $E_{0}(\mathrm{ATEM})$ & $E_{0}(V)$ & $E_{0}(N)$ & Error $(\%)$ & $E_{1}(\mathrm{ATEM})$ & $E_{1}(V)$ & $E_{1}(N)$ & Error $(\%)$ \\
\hline 0.1 & 1.023810 & 1.023910 & 1.023810 & 0.0000 & 3.70894 & 3.71064 & 3.70897 & 0.0008 \\
0.2 & 0.986535 & 0.986646 & 0.98654 & 0.0005 & 3.61702 & 3.61890 & 3.61704 & 0.0007 \\
0.3 & 0.948503 & 0.948629 & 0.948507 & 0.0004 & 3.52388 & 3.52596 & 3.52390 \\
0.4 & 0.909677 & 0.909820 & 0.909681 & 0.0004 & 3.42948 & 3.43179 & 3.42950 \\
0.5 & 0.870019 & 0.870181 & 0.870022 & 0.0004 & 3.33379 & 3.33636 & 3.33381 & 0.0007 \\
0.6 & 0.829486 & 0.829670 & 0.829488 & 0.0003 & 3.23678 & 3.23962 & 3.23679 \\
0.7 & 0.788033 & 0.788243 & 0.788035 & 0.0003 & 3.13839 & 3.14155 & 3.13840 \\
0.8 & 0.745612 & 0.745852 & 0.745613 & 0.0002 & 3.03858 & 3.04210 & 3.03859 \\
0.9 & 0.702171 & 0.702447 & 0.702172 & 0.0001 & 2.93733 & 2.94123 & 2.93733 \\
1 & 0.657656 & 0.657972 & 0.657656 & 0.0000 & 2.83456 & 2.83891 & 2.83456 & 0.0005 \\
2 & 0.137807 & 0.139170 & 0.137786 & 0.0152 & 1.71314 & 1.72629 & 1.71304 & 0.000003 \\
\hline
\end{tabular}

TABLE 3: Energy eigenvalues for the potential in (9) with different values of $\gamma \cdot E_{2,3}(V)$ and $E_{2,3}(N)$ show the values obtained by variational supersymmetric method and numerical integration, respectively. The percent errors for ATEM are also shown in the fifth and ninth columns.

\begin{tabular}{lcccccccc}
\hline$\gamma$ & $E_{2}(\mathrm{ATEM})$ & $E_{2}(V)$ & $E_{2}(N)$ & Error $(\%)$ & $E_{3}(\mathrm{ATEM})$ & $E_{3}(V)$ & $E_{3}(N)$ & Error $(\%)$ \\
\hline 0.1 & 7.33081 & 7.31384 & 7.33079 & 0.0002 & 11.48879 & 11.54258 & 11.48857 & 0.0019 \\
0.2 & 7.20494 & 7.18687 & 7.20491 & 0.0004 & 11.33161 & 11.38692 & 11.33136 & 0.0022 \\
0.3 & 7.07813 & 7.05889 & 7.07809 & 0.0005 & 11.17348 & 11.23045 & 11.17319 & 0.0026 \\
0.4 & 6.95037 & 6.92988 & 6.95033 & 0.0006 & 11.01439 & 11.07307 & 11.01406 & 0.0030 \\
0.5 & 6.82167 & 6.79984 & 6.82162 & 0.0008 & 10.85434 & 10.91477 & 10.85396 & 0.0035 \\
0.6 & 6.69203 & 6.66874 & 6.69197 & 0.0009 & 10.69332 & 10.75556 & 10.69288 & 0.0041 \\
0.7 & 6.56144 & 6.53660 & 6.56137 & 0.0010 & 10.53132 & 10.59547 & 10.53083 \\
0.8 & 6.42991 & 6.40340 & 6.42982 & 0.0014 & 10.36835 & 10.43448 & 10.36778 & 0.0047 \\
0.9 & 6.29744 & 6.26913 & 6.29734 & 0.0016 & 10.20439 & 10.27258 & 10.20375 & 0.0055 \\
1 & 6.16404 & 6.13379 & 6.16393 & 0.0018 & 10.03944 & 10.10978 & 10.03872 & 0.0072 \\
2 & 4.78291 & 4.72244 & 4.78245 & 0.0096 & 8.33516 & 8.43395 & 8.33293 & 0.0268 \\
\hline
\end{tabular}

TABLE 4: Comparison of the first few energy eigenvalues of potential in (17) with different values of $j$. The percent errors for ATEM are also shown in the fifth and tenth columns.

\begin{tabular}{|c|c|c|c|c|c|c|c|c|c|}
\hline$j$ & $n$ & $E_{\text {ATEM }}$ & $E_{\text {exact }}^{[31]}$ & Error (\%) & $j$ & $n$ & $E_{\text {ATEM }}$ & $E_{\text {exact }}^{[31]}$ & Error (\%) \\
\hline \multirow{7}{*}{0} & 0 & 0 & 0 & 0.0000 & \multirow{7}{*}{$1 / 2$} & 0 & -2.82843 & -2.82843 & 0.000 \\
\hline & 1 & 1.9354 & 1.9354 & 0.0000 & & 1 & -2.26507 & - & - \\
\hline & 2 & 6.2981 & 6.2985 & 0.0064 & & 2 & 2.83243 & 2.83843 & 0.1414 \\
\hline & 3 & 11.6869 & 11.6809 & 0.0514 & & 3 & 7.19836 & 7.19806 & 0.0042 \\
\hline & 4 & 18.1031 & 18.0426 & 0.3353 & & 4 & 13.0142 & 13.0093 & 0.0377 \\
\hline & \multirow[t]{2}{*}{5} & 24.7273 & 25.2546 & 2.0879 & & 5 & 19.6564 & 19.7215 & 0.3301 \\
\hline & & & & & & 6 & 26.6816 & 27.2400 & 2.0499 \\
\hline$j$ & $n$ & $E_{\text {ATEM }}$ & $E_{\text {exact }}^{[31]}$ & Error (\%) & $j$ & $n$ & $E_{\text {ATEM }}$ & $E_{\text {exact }}^{[31]}$ & Error (\%) \\
\hline \multirow{8}{*}{1} & 0 & -8 & -8 & 0.0000 & \multirow{8}{*}{$3 / 2$} & 0 & -15.0775 & - & - \\
\hline & 1 & 7.91736 & 7.91735 & 0.0001 & & 1 & -15.0686 & - & - \\
\hline & 2 & 0 & 0 & 0.0000 & & 2 & -3.55932 & - & - \\
\hline & 3 & 2.52034 & 2.52036 & 0.0008 & & 3 & -2.73313 & - & - \\
\hline & 4 & 8 & 8 & 0.0000 & & 4 & 3.55932 & - & - \\
\hline & 5 & 14.1105 & 14.1130 & 0.0177 & & 5 & 8.52713 & - & - \\
\hline & 6 & 21.1161 & 21.1575 & 0.1957 & & 6 & 15.0775 & - & - \\
\hline & 7 & 29.6605 & 28.9748 & 2.3665 & & 7 & 22.4392 & - & - \\
\hline
\end{tabular}


TABLE 5: Comparison of energy eigenvalues of the potential in (19) for $n=0,2,4$ states.

\begin{tabular}{cccc}
\hline$n$ & $E_{\text {ATEM }}$ & $E_{\text {WKB }}^{[6]}$ & $E_{\text {exact }}^{[32]}$ \\
\hline 0 & 3.7256160383 & 3.65904483 & 3.7256160383 \\
2 & 21.7457044301 & 21.745700933 & 21.7457044301 \\
4 & 44.3253501154 & 44.3253501144 & 44.3253501154 \\
\hline
\end{tabular}

\section{Conflict of Interests}

The authors declare that there is no conflict of interests regarding the publication of this paper.

\section{Acknowledgments}

The financial supports of Research Fund of Gaziantep University (BAP) and the Scientific and Technological Research Council of Turkey (TÜBİTAK) are acknowledged. The authors are also very much indebted to the referee(s) for useful comments and suggestions.

\section{References}

[1] Y. P. Varshni, "Eigenenergies and oscillator strengths for the Hulthén potential," Physical Review A, vol. 41, no. 9, pp. 4682$4689,1990$.

[2] M. A. Nunez, "Accurate computation of eigenfunctions for Schrödinger operators associated with Coulomb-type potentials," Physical Review A, vol. 47, no. 5, pp. 3620-3631, 1993.

[3] P. Matthys and H. de Meyer, "Dynamical-group approach to the Hulthén potential," Physical Review A, vol. 38, no. 3, pp. 11681171, 1988.

[4] C. Stubbins, "Bound states of the Hulthén and Yukawa potentials," Physical Review A, vol. 48, no. 1, pp. 220-227, 1993.

[5] Y. P. Varshni, "Relative convergences of the WKB and SWKB approximations," Journal of Physics A Mathematical and General, vol. 25, no. 21, pp. 5761-5777, 1992.

[6] G. A. Dobrovolsky and R. S. Tutik, "Regularization of the WKB integrals," Journal of Physics A: Mathematical and General, vol. 33, no. 37, pp. 6593-6599, 2000.

[7] A. Z. Tang and F. T. Chan, "Shifted $1 / \mathrm{N}$ expansion for the Hulthén potential," Physical Review A, vol. 35, no. 2, pp. 911-914, 1987.

[8] R. K. Roychoudhury and Y. P. Varshni, "Shifted 1/N expansion and exact solutions for the potential $V(r)=-Z / r+g r+\lambda r^{2}$," Journal of Physics A: Mathematical and General, vol. 21, no. 13, pp. 3025-3034, 1988.

[9] A. F. Nikiforov and V. B. Uvarov, Special Functions of Mathematical Physics, Birkhäuser, Basel, Switzerland, 1988.

[10] S. W. Qian, B. W. Huang, and Z. Y. Gu, "Supersymmetry and shape invariance of the effective screened potential," New Journal of Physics, vol. 4, p. 13, 2002.

[11] P. Maknikowski and A. Radosz, "Comment on "Bounces and the calculation of quantum tunnelling effects for the asymmetric double-well potential": [B. Zhou, J.-Q. Liang, F.-Ch. Pud, Phys. Lett. A 271 (2000) 26]," Physics Letters A, vol. 292, no. 4-5, pp. 300-302, 2002.

[12] B. Zhou, J. Q. Liang, and F. C. Pud, "Quantum tunneling for the asymmetric double-well potential at finite energy," Physics Letters A, vol. 281, no. 2-3, pp. 105-112, 2001.
[13] E. Paspalakis, "Physical interpretation of laser-induced suppression of quantum tunneling," Physics Letters A, vol. 261, no. 5-6, pp. 247-251, 1999.

[14] J. Chen, L. C. Kwek, and C. H. Oh, "Quartic anharmonic oscillator and non-hermiticity," Physical Review A: Atomic, Molecular, and Optical Physics, vol. 67, no. 1, Article ID 012101, 9 pages, 2003.

[15] F. Zhou, Z. Cao, and Q. Shen, "Energy splitting in symmetric double-well potentials," Physics Letters A, vol. 67, Article ID 062112, 2003.

[16] Y. He, Z. Cao, and Q. Shen, "Bound-state spectra for supersymmetric quantum mechanics," Physics Letters A, vol. 326, no. 5-6, pp. 315-321, 2004.

[17] A. Hutem and C. Sricheewin, "Ground-state energy eigenvalue calculation of the quantum mechanical well $V(x)=\left(\frac{1}{2}\right) k x^{2}+$ $\lambda x^{4}$ via analytical transfer matrix method," European Journal of Physics, vol. 29, no. 3, p. 577, 2008.

[18] R. Koç and S. Sayın, "Remarks on the solution of the positiondependent mass Schrödinger equation," Journal of Physics A: Mathematical and Theoretical, vol. 43, no. 45, Article ID 455203, 2010.

[19] Wolfram Research, "Mathematica, Version 8.0," Wolfram Research, Champaign, Ill, USA, 2010.

[20] O. Ozer, H. Koklu, and S. Resitoglu, "Application of the asymptotic Taylor expansion method to bistable potentials," Advances in Mathematical Physics, vol. 2013, Article ID 239254, 8 pages, 2013.

[21] C. S. Hsue and J. L. Chern, "Two-step approach to onedimensional anharmonic oscillators," Physical Review D, vol. 29, article 643, 1984.

[22] G. P. Flessas, R. R. Whitehead, and A. Rigas, "On the $\alpha \mathrm{x}^{2}+\beta \mathrm{x}^{4}$ interaction," Journal of Physics A, vol. 16, no. 1, p. 85, 1983.

[23] A. R. Bonham and S. L. Su, "Use of hellmann-feynman and hypervirial theorems to obtain anharmonic vibration-rotation expectation values and their application to gas diffraction," The Journal of Chemical Physics, vol. 45, p. 2827, 1996.

[24] M. Reed and B. Simon, Methods of Modern Mathematical Physics, IV Analysis of Operators, Academic Press, New York, NY, USA, 1978.

[25] C. Quigg and J. L. Rosner, "Quantum mechanics with applications to quarkonium," Physics Reports C, vol. 56, no. 4, pp. 167$235,1979$.

[26] A. Khare and Y. P. Varshni, "Is shape invariance also necessary for lowest order supersymmetric WKB to be exact?" Physics Letters A, vol. 142, no. 1, pp. 1-4, 1989.

[27] G. R. P. Borges, A. de Souza Dutra, E. Drigo, and J. R. Ruggiero, "Variational method for excited states from supersymmetric techniques," Canadian Journal of Physics, vol. 81, no. 11, pp. 12831291, 2003.

[28] G. Harvey and J. Tobochnik, An Introduction to Computer Simulation Methods: Applications to Physical Systems, AddisonWesley, Reading, Mass, USA, 2nd edition, 1996.

[29] A. V. Turbiner, "Quasi-exactly-solvable problems and $\operatorname{sl}(2)$ algebra," Communications in Mathematical Physics, vol. 118, no. 3, pp. 467-474, 1988.

[30] M. A. Shifman, "New findings in quantum mechanics (partial algebraization of the spectral problem)," International Journal of Modern Physics A, vol. 4, no. 12, pp. 2897-2952, 1989.

[31] P. Roy, R. Roychoudhury, and Y. P. Varshni, "On the application of supersymmetric WKB (Wentzel-Kramers-Brillouin) 
method to quasi-exactly solvable problems," Canadian Journal of Physics, vol. 69, no. 10, pp. 1261-1263, 1991.

[32] R. N. Chaudhuri and M. Mondal, "Eigenvalues of anharmonic oscillators and the perturbed Coulomb problem in $\mathrm{N}$ dimensional space," Physical Review A, vol. 52, no. 3, pp. 18501856, 1995.

[33] V. Jelic and F. Marsiglio, "The double-well potential in quantum mechanics: a simple, numerically exact formulation," European Journal of Physics, vol. 33, no. 6, pp. 1651-1666, 2012. 


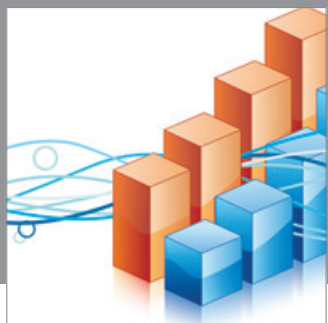

Advances in

Operations Research

mansans

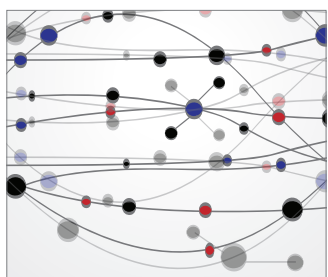

The Scientific World Journal
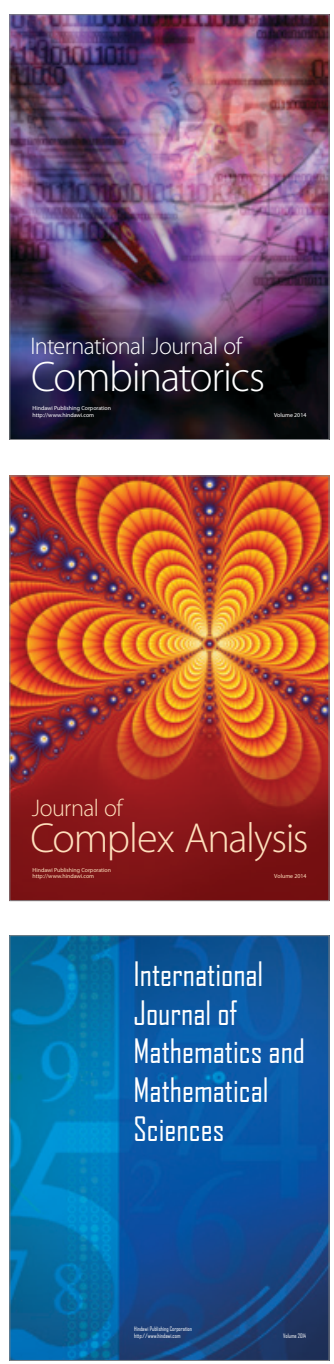
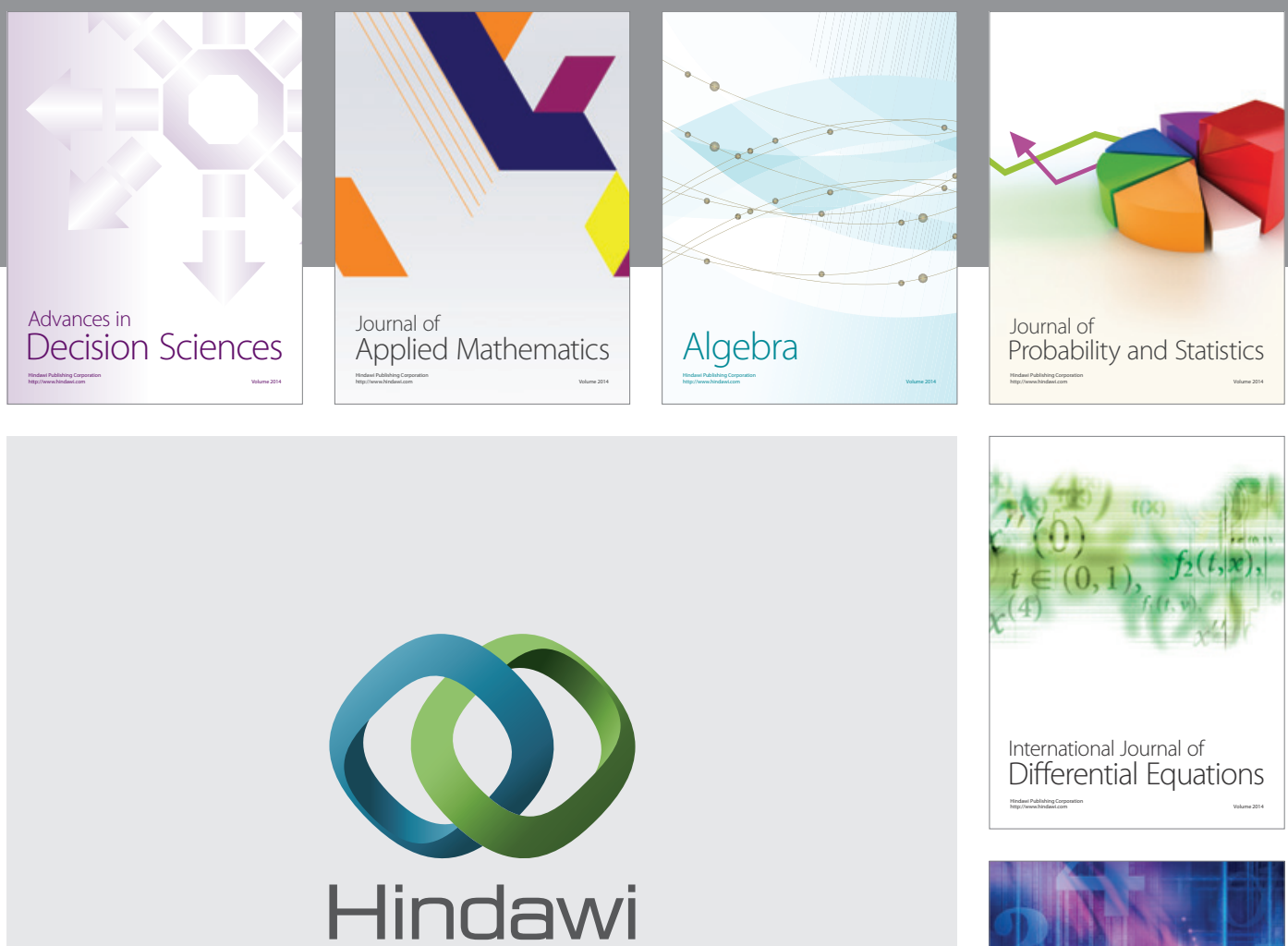

Submit your manuscripts at http://www.hindawi.com
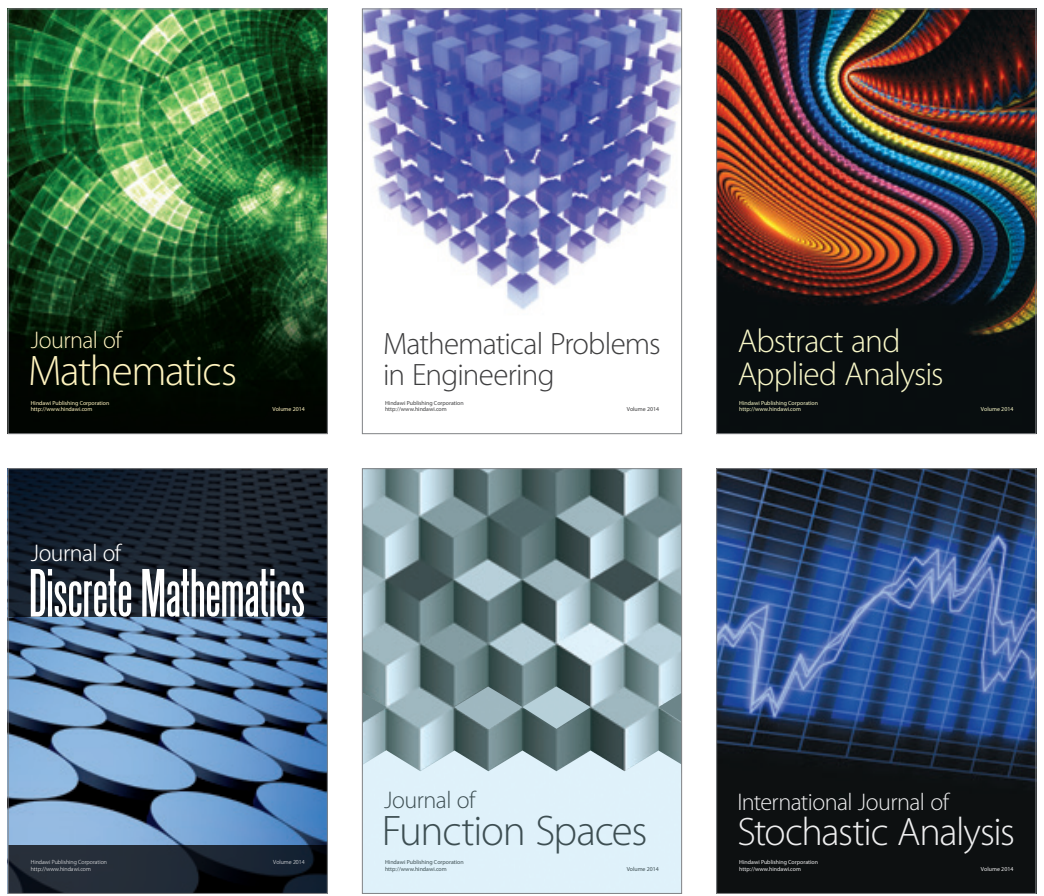

Journal of

Function Spaces

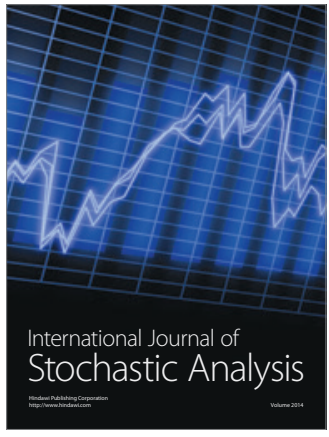

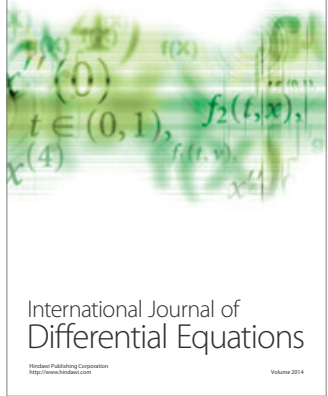
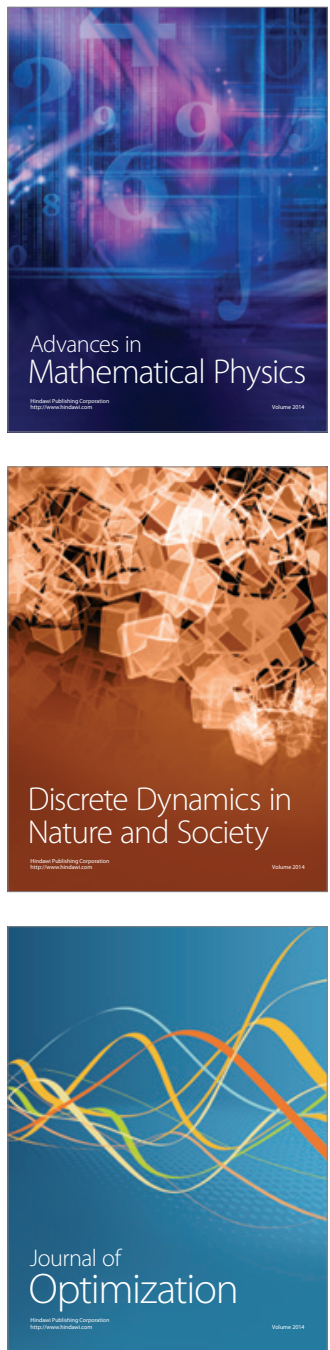